\title{
UM INSTRUMENTO PARA MEDIDA DE POTENCIAL MATRICIAL NOS SOLOS SEM OCORRÊNCIA DE CAVITAÇÃO ${ }^{(1)}$
}

\author{
Abdoul Aziz Diene ${ }^{(2)}$ \& Claudio Fernando Mahler ${ }^{(3)}$ \\ RESUMO
}

\begin{abstract}
A tensão nos solos tem sido vista pelos cientistas do solo como um aspecto de importância crescente sendo bastante usada como informação relevante na agricultura desde o início do século passado. Seu conhecimento permite melhores intervenções antrópicas não só em aspectos relativos à vegetação e a agricultura, mas também no que concerne à estabilidade de um talude. Para medir a tensão no solo tensiometros cada vez mais sofisticados tem sido desenvolvidos. É apresentado neste trabalho o estado da arte da evolução dos tensiômetros, além do desenvolvimento de um deles, o qual mede valores de tensão de até pelo menos $1.500 \mathrm{kPa}$. Diversos protótipos foram preparados. Observou-se que se comportam diferentemente de acordo com as características de seus componentes, sem grande influência nos resultados nos domínios de leitura possíveis em cada caso. Assim, os efeitos de tipo de transdutor, tamanho do reservatório de água, dimensões e permeabilidade ao ar da pedra porosa foram analisados, monitorando-os quando instalados em lisímetros, junto com outros equipamentos, como equitensiômetros e TDRs. Os resultados mostraram que o protótipo pode ser usado in situ e em laboratório, sendo facilmente adaptável para uso em oedômetro ou aparelho de ensaio triaxial. Comentários sobre o papel das forças atrativas de Van der Waals na cavitação do sistema são também apresentados, esclarecendo-se os procedimentos para deslocamento do ponto de cavitação para limites superiores com a diminuição dos possíveis núcleos de gás no sistema.
\end{abstract}

Termos de indexação: tensiometria, solos não saturados.

\section{SUMMARY: A TOOL FOR MEASUREMENT OF SOIL MATRIX POTENTIAL WITHOUTCAVITATION}

Soil suction has been regarded by soil scientists as an aspect of increasing importance and has been used as an important source of information in agriculture since early last century. Its knowledge helps improve human actions not only in aspects relating to vegetation and agriculture, but also concerning slope stability. Increasingly sophisticated

\footnotetext{
(1) Recebido para publicação janeiro de 2006 e aprovado em julho de 2007.

${ }^{(2)}$ Doutorando no Centro de Tecnologia, Universidade Federal do Rio de Janeiro - COPPE/UFRJ. Bloco B Caixa Postal 68506, CEP 21945-970 Rio de Janeiro (RJ). Bolsista da CAPES. E-mail: abdoulazizdiene@yahoo.com

${ }^{(3)}$ Professor do Centro de Tecnologia, COPPE/UFRJ. E-mail: cfmahler@acd.ufrj.br
} 


\begin{abstract}
tensiometers have been designed to measure soil suction. This study describes the state of the art in the evolution of tensiometers, aside from the development of a tensiometer which measures stress values of at least $1.500 \mathrm{kPa}$. Various prototypes were developed. These devices differed in the characteristics of their components, but performed rather similarly in the results in the possible reading ranges in each case. The effects of the type of transducer, size of the water tank, dimensions and permeability to air of porous stone were analyzed and monitored, installed in lysimeters or together with other equipment such as equitensiometers and TDRs. Results showed that the prototype may be used in situ and in laboratory and is readily adaptable for use in an air oedometer or triaxial apparatus. The role of Van der Waals forces in the cavitation of the system was also discussed, explaining the procedures for shifting the point of cavitation to upper limits aiming at a reduction of possible gas nuclei in the system.
\end{abstract}

Index terms: non-saturated soils, soil matrix potential, tensiometry.

\section{INTRODUÇÃO}

\section{Os tensiômetros de tensão elevada (estado da arte)}

Tabor (1979) demonstrou teoricamente que a resistência à tração da água fica por volta de $-500.000 \mathrm{kPa}$ (Ridley \& Burland, 1993). Partindo dessa tese, Ridley (1993) e Ridley \& Burland (1993) apresentaram um equipamento (Figura 1) capaz de medir tensões da ordem de $1.000 \mathrm{kPa}$.

König et al. (1994) usaram um tensiômetro para medida de poropressão em centrífuga: o Druck PDCR81 (Figura 2). Usando um dispositivo de domínio de medição amplo, com uma pedra de 15 bar de entrada de ar, saturada a uma pressão de $2.000 \mathrm{kPa}$, Ridley (1995) foi capaz de medir tensões próximas a $1.370 \mathrm{kPa}$.

Ridley \& Burland (1995) apresentaram um outro protótipo, com base no princípio de maximização da tensão sustentável em tensiômetros para medir tensões elevadas em solo. Este tensiômetro possibilitou medidas in situ de tensão da ordem de $1.500 \mathrm{kPa}$. O equipamento consiste de um diafragma com straingauge integrado e de um elemento poroso em cerâmica

Pedra cerâmica de 15 bar

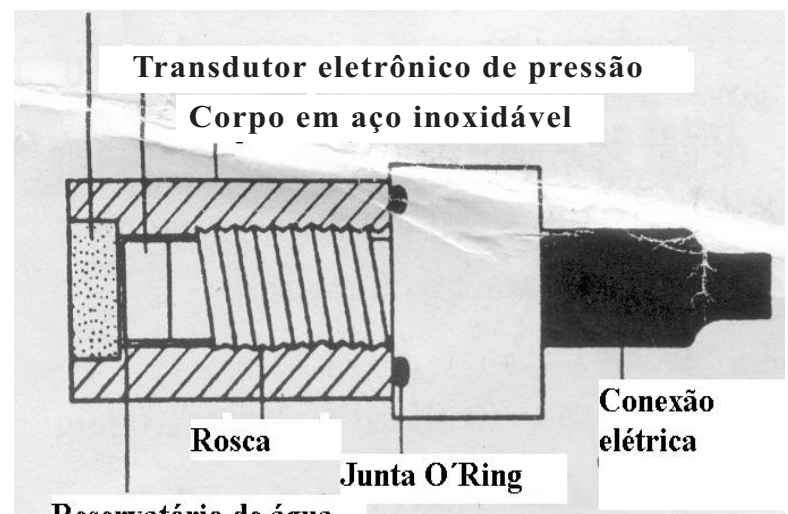

Reservatório de água

Figura 1. Tensiômetro desenvolvido no Imperial College - Inglaterra. (Ridley \& Burland, 1993) selado com valor de $1.500 \mathrm{kPa}$ de pressão de entrada de ar, saturada a uma pressão de $2.000 \mathrm{kPa}$ (Figura 3).

Guan \& Fredlund (1997) apresentaram um tensiômetro capaz de medir valores de tensão em solos superiores a $1.250 \mathrm{kPa}$ quando saturado sob seis ciclos de pressão entre 12.000 e $85 \mathrm{kPa}$. A pedra cerâmica de alto valor de entrada de ar é selada pela metade numa caixa destacável do dispositivo. Montada dentro da água, a outra metade da caixa atua como elemento de compressão, ajustando e selando o transdutor comercial no reservatório de água (Figura 4).

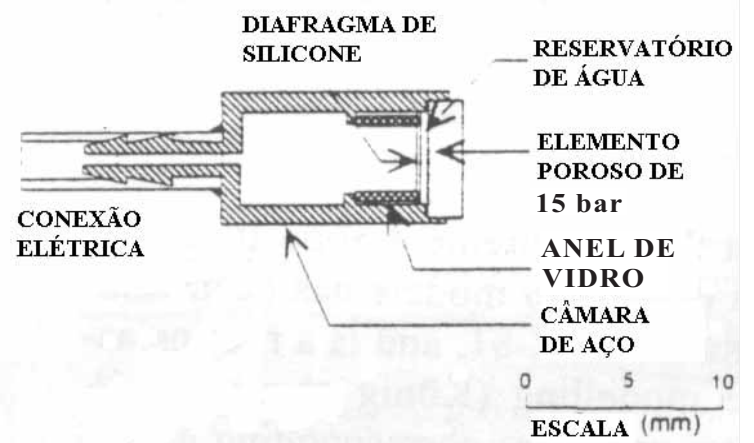

Figura 2. Tensiômetro desenvolvido por König et al. (1994).

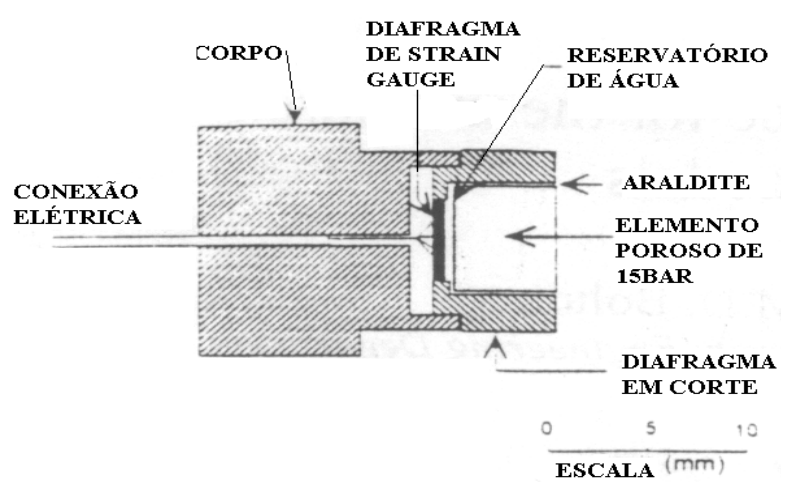

Figura 3. Tensiômetro desenvolvido no Imperial College - Inglaterra. (Ridley \& Burland, 1995) 
Pacheco (2001) e Mahler et al. (2002) desenvolveram um tensiômetro de baixo custo (Figura 5), com medições maiores do que $300 \mathrm{kPa}$, sem que ocorresse a cavitação.

Take \& Bolton (2002) conceberam novos instrumentos mais robustos para medição de poropressão negativa em centrífuga. Para isso, optaram por desenvolver três protótipos, apresentando algumas particularidades de formato para cada um. Em comum, os protótipos foram equipados com um transdutor Entran EPB de $700 \mathrm{kPa}$ e uma pedra porosa de $1.500 \mathrm{kPa}$ (Figura 6).

Tarantino \& Mongiovi (2002) apresentaram um novo tensiômetro, parecido com o de Ridley \& Burland (1995), mas com várias modificações no diâmetro e espessura do diafragma, no tamanho do reservatório de água e no fechamento da abertura anular entre a pedra porosa e o corpo do tensiômetro (Figura 7). Este novo equipamento mediu tensões acima de $1.000 \mathrm{kPa}$

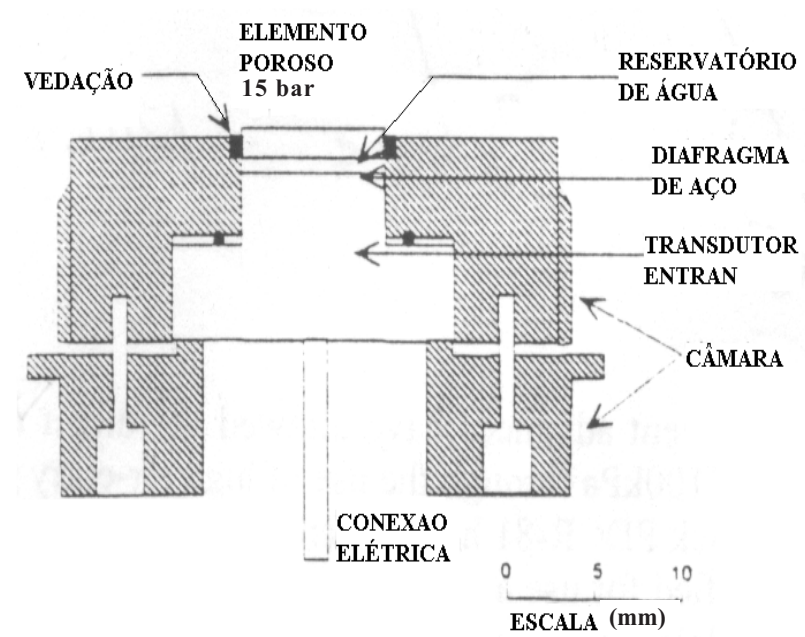

Figura 4. Tensiômetro desenvolvido na University of Saskatchewan - Canadá. (Guan \& Fredlund, 1997)

durante um tempo superior a 15 dias e atingiu a máxima tensão de $2.000 \mathrm{kPa}$.

Todos esses protótipos conseguiram leituras de tensão no solo sem ocorrência de cavitação acima de $100 \mathrm{kPa}$. Dentre os vários modelos que explicam o fenômeno de cavitação de água sujeita à tensão, um dos mais aceitos é o proposto por Harvey et al. (1944): "assume-se que a cavitação origina-se dos núcleos de gases não dissolvidos que existem nos interstícios das paredes do reservatório em vez das cavidades livres do líquido"; isso pelo fato de que o núcleo esférico de gás livre é geralmente instável e tende a ir para o interior do líquido. Ao contrário, o núcleo de gás nas cavidades das paredes do contêiner pode ficar indissolúvel mesmo sob alta pressão de água. Os ensaios de tração da água foram feitos, usando vidro ou aço inox em tubo de Berthelot.

Para inibir o fenômeno de cavitação nos equipamentos, várias soluções foram propostas por diversos autores, que se resumem a procedimentos aplicados na montagem ou na saturação e, ou, calibração deles. Dessas propostas, surgiram algumas conclusões referentes a como evitar a cavitação. Ridley (1993) considerou que a máxima tensão sustentável pelo tensiômetro é também função do valor de entrada de ar do elemento poroso. "Se a diferença de pressão entre o reservatório e a medida no solo exceder o valor da entrada de ar, este pode ser 'puxado' de dentro do reservatório de água; mudanças de pressões externas que atuam no dispositivo vão resultar na expansão ou contração das bolhas do ar e a pressão medida é potencialmente destacada da realidade". Marinho \& Chandler (1995) sugeriram o uso de um volume pequeno de água no sistema de medição de tensão como responsável para que não aconteça cavitação e que o volume mínimo possível está limitado pelo deslocamento do diafragma do transdutor. A pré-pressurização da água tem sido considerada essencial para saturação dos tensiômetros; contudo, algumas diferenças existem entre os métodos de pré-pressurização. (a)

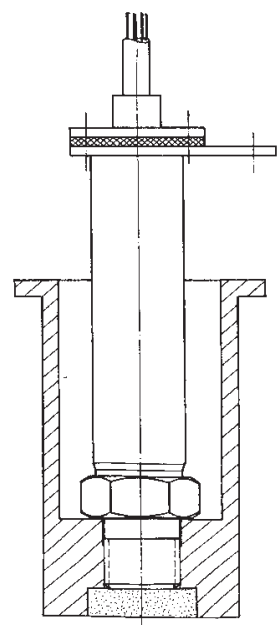

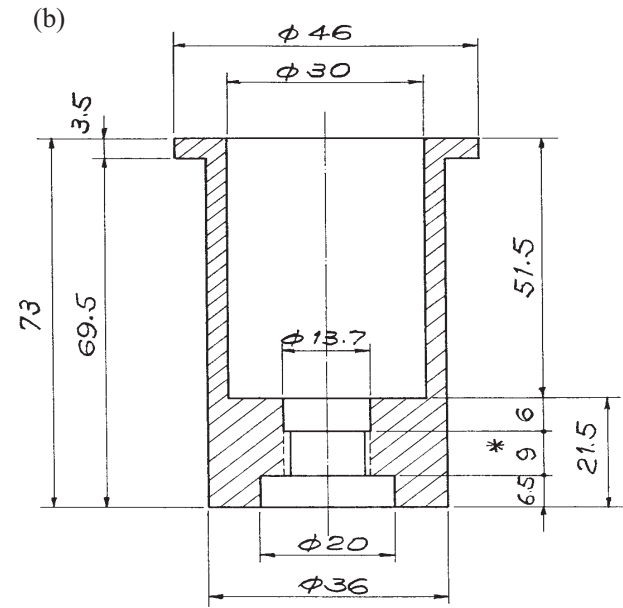

Figura 5. Tensiômetro desenvolvido na COPPE - UFRJ (a e b). (Pacheco, 2001; Mahler et al., 2002) 

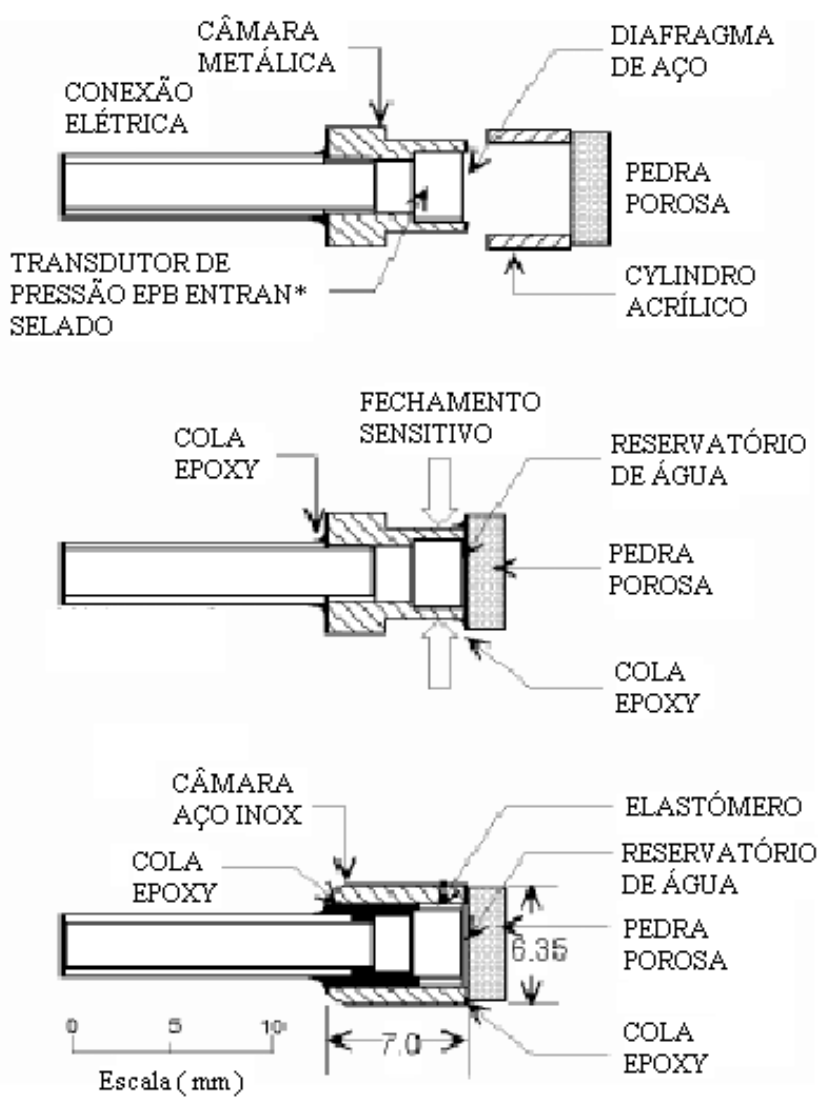

Figura 6. Tensiômetro desenvolvido na Cambridge University - Inglaterra. Take \& Bolton (2002)

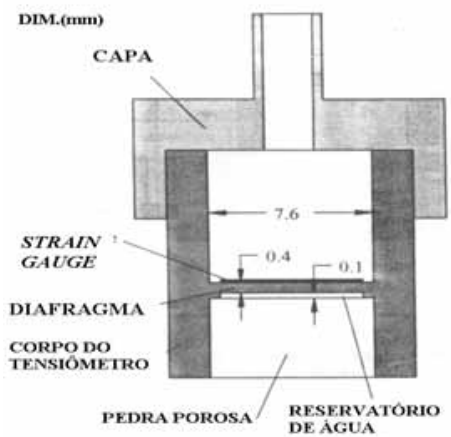

Figura 7. Tensiômetro desenvolvido na Universitá di Trento - Itália. (Tarantino \&. Mongiovi, 2002)

Guan \& Fredlund (1997) aplicam varias prépressurizações em ciclos que começam sob um vácuo de $85 \mathrm{kPa}$, seguido por pressões maiores, que vão até $12.000 \mathrm{kPa}$. Tem sido sugerido que a tensão de rompimento é afetada preliminarmente pelo número de ciclos e pela magnitude da pressão positiva aplicada (Guan et al., 1998). Ridley \& Burland (1999) afirmaram que o processo da pré-pressurização é menos importante; enquanto isso, a saturação inicial da pedra porosa tem um papel mais importante. Esses autores propõem que se sature o tensiômetro pela aplicação de uma pressão constante de $4.000 \mathrm{kPa}$, que é mantida por pelo menos $24 \mathrm{~h}$. Tarantino et al. (2000) mediram tensões superiores ao valor de entrada de ar do elemento poroso sem que houvesse cavitação e concluíram que a "cavitação pode ocorrer antes da equalização do sistema solo-equipamento, causando a interrupção do teste e a pré-saturação do instrumento; o conhecimento das condições que lidam com a cavitação é, portanto, essencialmente baseado na otimização do design do instrumento".

A cavitação não ocorrerá caso o sistema esteja livre de núcleos de cavitação, o que significa, resumidamente, o uso de água deaerada pura e limpa, superfícies extremamente lisas e limpas, submissão do sistema ao vácuo, aplicação cíclica de pressões positivas e negativas e pré-pressurização do sistema a altas pressões para dissolver o ar livre.

\section{MATERIAL E MÉTODOS}

Um tensiômetro é composto basicamente por uma pedra porosa, um corpo de contenção desta pedra e um transdutor ou equivalente, que transforma as variações de tensão no solo em leitura de pressões.

Os problemas referentes à cavitação - que, em geral, ocorrem a aproximadamente uma tensão de $80 \mathrm{kPa}$ - são muito comuns com tensiômetros de estrutura de montagem simples. Esses problemas foram superados nessa nova forma de construção de tensiômetros, com refinamentos construtivos relevantes, pelas seguintes ações: uso de pedra porosa com elevado valor de pressão de entrada de ar; uso de água deaerada durante todo o processo de saturação da pedra porosa e sua montagem; uso do material acrílico na construção do corpo do tensiômetro devido à sua superfície lisa, fazendo com que não haja possibilidade de presença de microbolha de ar durante o processo de saturação do sistema; ciclagem de pressões de carga e descarga no processo de calibração do equipamento; e especificações técnicas e características do transdutor escolhido.

O equipamento aqui apresentado também é composto apenas destes três elementos - pedra porosa, transdutor e corpo de acrílico - e tem algumas semelhanças com o equipamento de Ridley (1993) e Ridley \& Burland (1993). Esse sistema é simplificado em termos construtivos, sendo fabricado de forma a se adaptar às dimensões originais da pedra porosa e do transdutor que se deseja empregar em cada caso específico (Figuras 8 e 9).

As especificações das capsulas porosas utilizadas estão apresentadas no quadro 1 ; na figura 10 pode ser vista a estrutura da pedra do tipo porosa. As pedras porosas de cerâmica são naturalmente molháveis e hidrofílicas, geralmente inertes, podendo ser produzidas com grande consistência e uniformidade da estrutura de seus poros. 

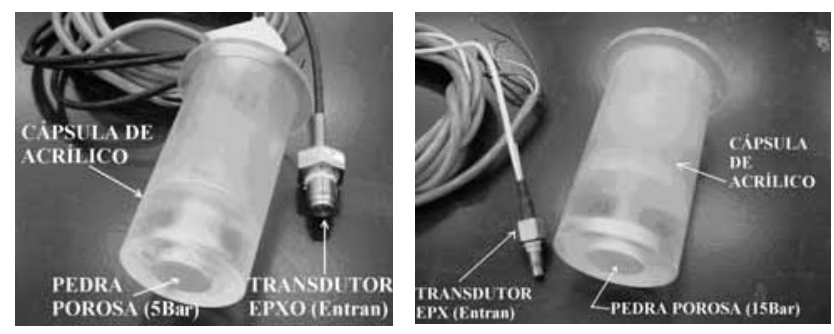

Figura 8. Tensiômetros desenvolvidos - Tense EPXO e Tense EPX. Diene (2004)

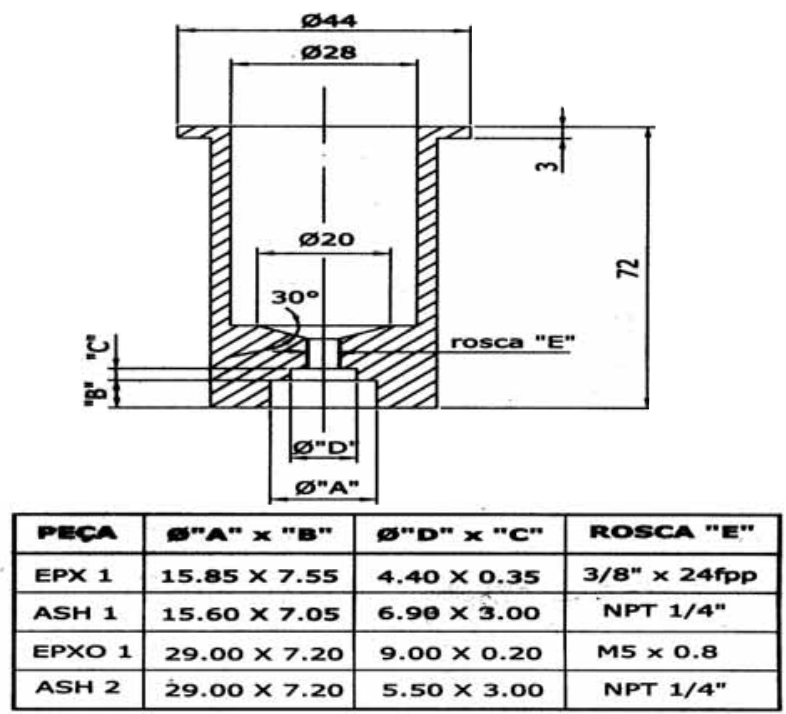

DIMENSÕES, $\mathrm{mm}$

Figura 9. Croquis do protótipo da cápsula de acrílico, com suas diferentes dimensões. Diene (2004)
No desenvolvimento dos protótipos de tensiômetro, foram empregados os transdutores e pedras porosas (Quadro 2).

Nos estudos foram empregados, junto aos tensiômetros, outros equipamentos servindo de controle e monitoramento, como os equitensiômetros e TDR, para monitoramento da umidade.

O Tensiômetro de Equivalência, ou Equitensiômetro EQ2, é composto por uma sonda Theta e um corpo de acrílico (Figura 11), e utiliza a mais recente geração de sensores de umidade. O sensor do equitensiômetro consiste da sonda Theta embutida em um material poroso especialmente projetado. O conteúdo de água deste material entra em equilíbrio com o potencial matricial do solo envolvido, onde é detectada pela sonda Theta, quando absorvida. Seu funcionamento baseia-se na equivalência do potencial matricial entre o material do solo e o corpo do instrumento. A princí-
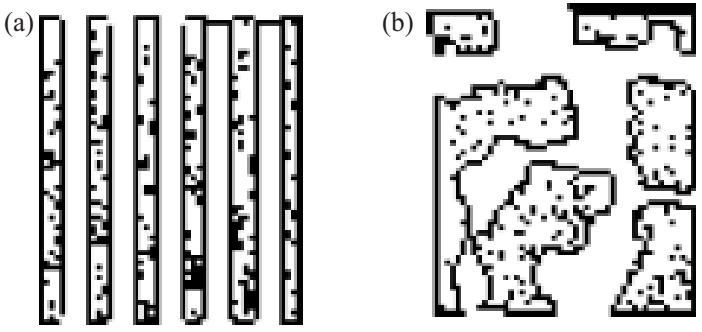

Figura 10. Estrutura de pedra porosa aberta - vista em corte longitudinal (a) e transversal (b) (Soil Moisture®).

Quadro 1. Especificação técnica das pedras porosas utilizadas, fornecidas pelo fabricante (Soil Moisture ${ }^{\circledR}$ )

\begin{tabular}{|c|c|c|c|c|c|}
\hline $\begin{array}{c}\text { Valor de pressão } \\
\text { de entrada } \\
\text { de ar }\end{array}$ & $\begin{array}{c}\text { Pressão de } \\
\text { borbulhamento }\end{array}$ & $\begin{array}{l}\text { Porosidade } \\
\text { aproximada }\end{array}$ & $\begin{array}{c}\text { Condutividade } \\
\text { hidraúlica } \\
\text { saturada }\end{array}$ & $\begin{array}{l}\text { Máximo } \\
\text { tamanho } \\
\text { de poros }\end{array}$ & $\begin{array}{c}\text { Escoamento } \\
\text { direto } \\
\text { "1/4-inch plate" }\end{array}$ \\
\hline & $\mathrm{kPa}$ & $\%$ vol & $\mathrm{cm} \mathrm{s}^{-1}$ & $\mu \mathrm{m}$ & $\mathrm{mL} \mathrm{h}^{-1} \mathrm{~cm}^{-2} / 101 \mathrm{kPa}$ \\
\hline $\begin{array}{l}500 \mathrm{kPa} \\
\text { Fluxo-padrão }\end{array}$ & 552 & $31 \%$ & $1,21 \times 10^{-7}$ & 0,5 & 0,7 \\
\hline $\begin{array}{l}1.500 \mathrm{kPa} \\
\text { Fluxo-padrão }\end{array}$ & 1.520 & $32 \%$ & $2,59 \times 10^{-9}$ & 0,16 & 0,015 \\
\hline
\end{tabular}

Quadro 2. Dimensões e características dos componentes dos tensiômetros usados nos ensaios (Diene, 2004)

\begin{tabular}{|c|c|c|c|c|}
\hline Tensiômetro & \multicolumn{2}{|c|}{$\begin{array}{c}\text { TENSE-EPX.1 } \\
\text { DIAM./ESP. }\end{array}$} & \multicolumn{2}{|c|}{$\begin{array}{c}\text { TENSE-EPXO.1 } \\
\text { DIAM./ESP. }\end{array}$} \\
\hline Pedra & 15,85 & 7,55 & 29 & 7,20 \\
\hline Pressão de entrada de ar $(\mathrm{kPa})$ & & & & 0 \\
\hline
\end{tabular}


pio, a resposta do instrumento $\mathrm{EQ} 2$ varia numa faixa de 0 a $-1.000 \mathrm{kPa}$, e sua melhor exatidão fica entre $100 \mathrm{kPa}$ e $-1.000 \mathrm{kPa}$, com $\pm 5 \%$ de erro. A sua exatidão de leitura em tensões de 0 a $100 \mathrm{kPa}$ é de $10 \mathrm{kPa}$.

A sonda Thetaprobe ML2 (Delta-T Devices, Cambridge, UK) é um instrumento de fabricação inglesa que mensura diretamente a umidade do solo, correspondendo ao conteúdo de umidade volumétrica, $\theta_{\mathrm{v}}$, por meio do princípio de geração de ondas que liberam um pulso eletromagnético para um conjunto de hastes sendo a reflexão medida no domínio do tempo (TDR).

O processo de saturação e calibração compreendeu as seguintes ações e equipamentos (Figura 13):

- Verificação da incorporação da pedra porosa ao corpo de acrílico, através de aplicação de três ciclos de pressão de água, para verificar a estanqüeidade do contato (pedra porosa-acrílico).

- A saturação da pedra porosa é feita com a aplicação de vácuo na câmara de calibração, sem que esta contenha água no seu interior por um período superior a $15 \mathrm{~h}$. Posteriormente, faz-se a entrada de água deaerada na câmara (mantendo-se o vácuo aplicado) até que ela cubra com certa folga o elemento poroso. Mantém-se o vácuo no sistema por mais duas horas.

- Transferência do conjunto para a câmara de calibração/saturação e aplicação de ciclos de pressões variando de zero até $600 \mathrm{kPa}$, seguindo o valor da pressão de borbulhamento da pedra usada, para gerar fluxo de água na pedra e remover eventuais bolhas.

- Instalação do transdutor de pressão no sistema por aparafusamento no corpo acrílico dentro de água deaerada e reaplicação de vácuo no sistema durante três a quatro horas.

- Calibração do protótipo por meio de aplicação de pressão positiva num sistema de potes de Hg com três estágios de carga e descarga, com valores de pressão de água até atingir a linearizaçao da curva de calibração (estes valores de pressão de água variam segundo o protótipo instalado).

Observou-se que durante a calibração dos equipamentos o tempo de resposta dos tensiômetros equipados com transdutores com membrana facial $\left(\right.$ EPX e EPXO $<$ Entran $^{\circledR}>$ ) é imediato (em menos de 30 s), após aplicação da carga de pressão. Com relação à sensibilidade, os transdutores variaram

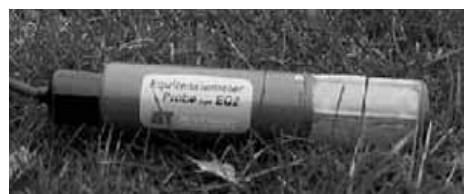

Figura 11. Esquema do Equitensiômetro EQ2 (Delta - T Devices). UK (2000)

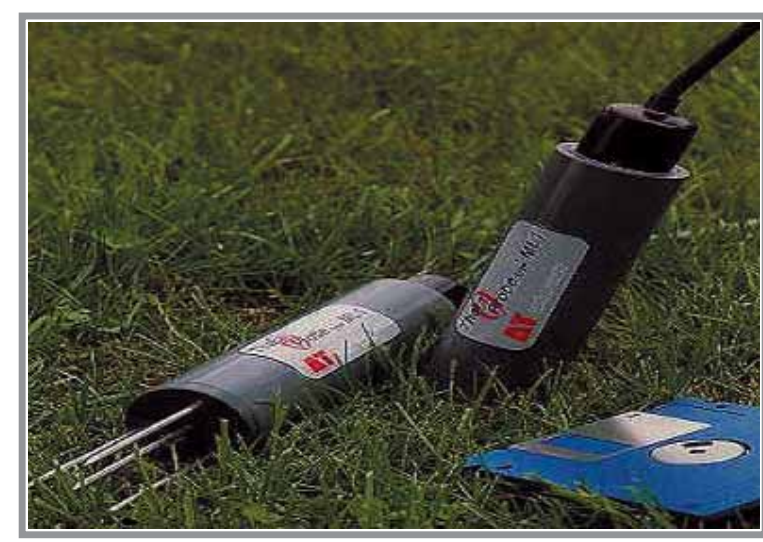

Figura 12. TDR Sonda Theta ML2 (Delta - T Devices). UK (2000)

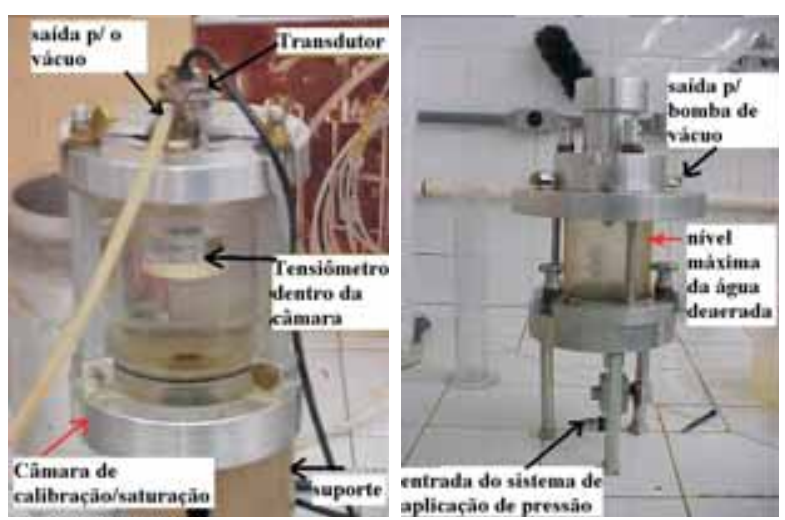

Figura 13. Câmara de calibração e câmara de saturação da pedra porosa. Diene (2004)

adequadamente dentro de suas frações de calibração; portanto, não se registrou notável diferença de comportamento quanto à histerese das suas membranas.

Os equipamentos foram ensaiados em dois minilisímetros de drenagem sem nível freático, com base no modelo de lisímetros de Thornthwaite, pela sua simplicidade, seu baixo custo e sua eficácia em determinar balanço hídrico em períodos longos de meses, cujas dimensões, em centímetros, são de $160 \times 60 \times 60$. Os equipamentos foram instalados nas profundidades de 15 e $30 \mathrm{~cm}$.

O solo utilizado nos lisímetros tem sua classificação pedológica, definindo-se como: Cambissolo Hálico Tb distrófico argissólico textura média A proeminente, hipodistrófico caulinítico hipoférrico muito profundo, ácido fase floresta tropical subperenifólia relevo montanhoso (Quadro 3). Não foi colocada nenhuma cobertura vegetal nos lisímetros, e o ensaio foi feito em ambiente fechado.

A classificação geotécnica do solo usado nesses tanques, pela sua origem, indica que ele é residual maduro (Figura 14). 
Quadro 3. Caracterização físico-química do solo I do tanque B (Laps-Embrapa/Cnps)

\begin{tabular}{|c|c|c|c|c|c|c|c|c|c|c|c|c|c|c|}
\hline \multirow[b]{2}{*}{ Hor. } & \multirow[b]{2}{*}{ Prof. } & \multicolumn{3}{|c|}{ Fração da amostra total } & \multicolumn{4}{|c|}{ Composição granulométrica } & \multirow[b]{2}{*}{$\begin{array}{c}\text { Argila } \\
\text { dispersa } \\
\text { em água }\end{array}$} & \multirow[b]{2}{*}{$\begin{array}{c}\text { Índice de } \\
\text { floculação }\end{array}$} & \multirow[b]{2}{*}{$\begin{array}{c}\text { Relação } \\
\text { silte/ } \\
\text { argila }\end{array}$} & \multicolumn{2}{|c|}{ Densidade } & \multirow[b]{2}{*}{$\begin{array}{l}\text { Porosi- } \\
\text { dade }\end{array}$} \\
\hline & & $\begin{array}{l}\text { Calhaus } \\
>20 \mathrm{~mm}\end{array}$ & $\begin{array}{c}\text { Cascalho } \\
20 \mathrm{a} \\
2 \mathrm{~mm}\end{array}$ & $\begin{array}{c}\text { Terra } \\
\text { fina }\end{array}$ & $\begin{array}{l}\text { Areia } \\
\text { grossa }\end{array}$ & $\begin{array}{c}\text { Areia } \\
\text { fina }\end{array}$ & Silte & Argila & & & & Solo & Partícula & \\
\hline
\end{tabular}

\begin{tabular}{|c|c|c|c|c|c|c|c|c|c|c|c|c|c|c|}
\hline \multirow[b]{2}{*}{ Ap } & \multirow{2}{*}{$\begin{array}{l}\mathrm{cm} \\
0-18\end{array}$} & \multicolumn{8}{|c|}{$\mathrm{g} \mathrm{kg}^{-1}$} & \multicolumn{2}{|l|}{$\%$} & \multicolumn{2}{|c|}{$-\mathrm{g} \mathrm{cm}^{-3}$} & \multirow{2}{*}{$\begin{array}{l}\% \\
47\end{array}$} \\
\hline & & 0 & 45 & 955 & 454 & 109 & 201 & 236 & 128 & 46 & 0,85 & 1,4 & 2,7 & \\
\hline $\mathrm{AB}$ & $18-36$ & 0 & 25 & 975 & 479 & 97 & 201 & 223 & 122 & 45 & 0,9 & 1,4 & 2,6 & 47 \\
\hline A2 & $36-60$ & 0 & 32 & 968 & 412 & 130 & 193 & 265 & 204 & 23 & 0,73 & 1,3 & 2,6 & 51 \\
\hline
\end{tabular}

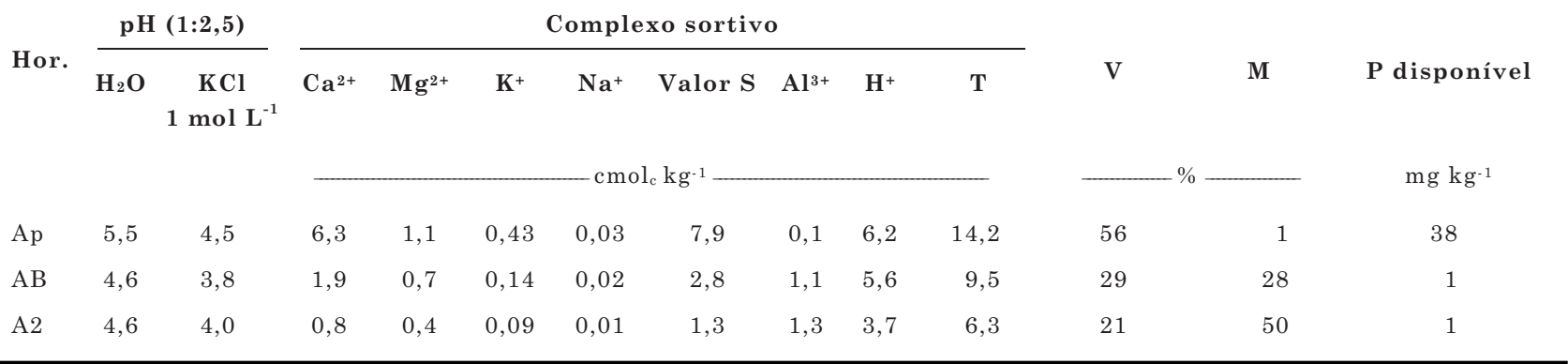

Foram traçadas as curvas de retenção de umidade, feitas a partir das amostras coletadas no campo em diferentes profundidades (Figura 14).

\section{RESULTADOS}

Nas figuras 15 e 16 são apresentados os resultados obtidos nos ensaios de laboratório em lisímetros (Diene, 2004), comparados com os de outros tensiômetros automatizados e sistemas de medição de tensão diferentes, como o equitensiômetro.

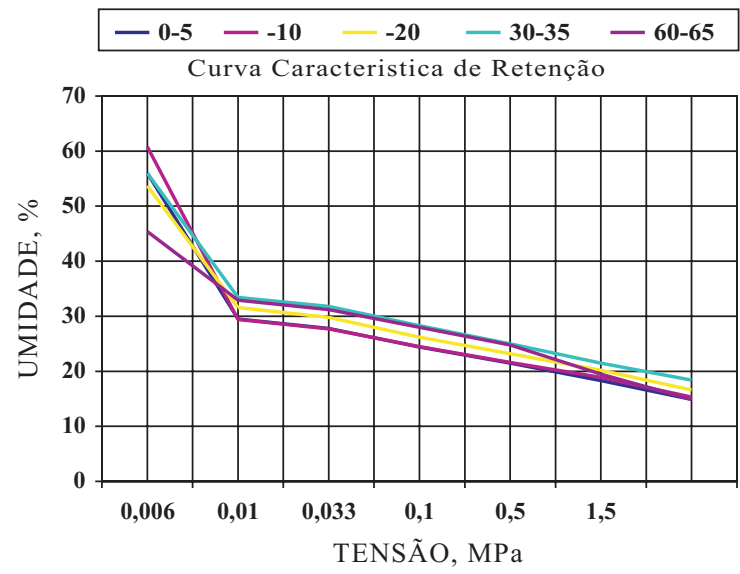

Figura 14. Curvas características de retenção de umidade do solo do tanque B (Laps-Embrapa/ CNPS). Diene (2004).
O tensiômetro TENSE-EPXO1 mediu valores acurados de tensão no solo de forma contínua até valores superiores ao valor de pressão de entrada de ar da pedra de $500 \mathrm{kPa}$ nele acoplada.

Os tensiômetros TENSE-EPX1 e TENSE-EPXO1 instalados a $15 \mathrm{~cm}$ de profundidade mediram valores de tensão aproximadamente similares. O tensiômetro mediu valores até $-1.465 \mathrm{kPa}, \mathrm{e}$, quando correlacionado com o TENSE-EPXO1, apresentou diferença de pressão decrescente, da ordem de 15 a $25 \mathrm{kPa}$.

O EQ2-B15 mediu durante todo o ensaio valores acima daqueles medidos pelos tensiômetros TENSE EPXO 1 e EPX 1, numa faixa de 0 a $100 \mathrm{kPa}$; no entanto, numa média de $49 \mathrm{kPa}$, a tendência da curva manteve-se conforme o esperado, acompanhando na mesma escala as variações dos dois outros equipamentos.

As curvas características do solo no tanque $\mathrm{B}$, assim representadas pelos dados mensurados pelo tensiômetro TENSE-EPX1 e equitensiômetro EQ-B15, são similares às curvas características determinadas no laboratório da Embrapa.

Na figura 17 é apresentado o desempenho do TDR durante os ensaios. A faixa de umidade mensurada variou entre 50 e $12 \%$. Os dados mensurados pela sonda TDR durante os ensaios após a saturação e drenagem, e nos períodos posteriores, foram satisfatórios, quando comparados aos fornecidos pela curva característica do solo in situ. 


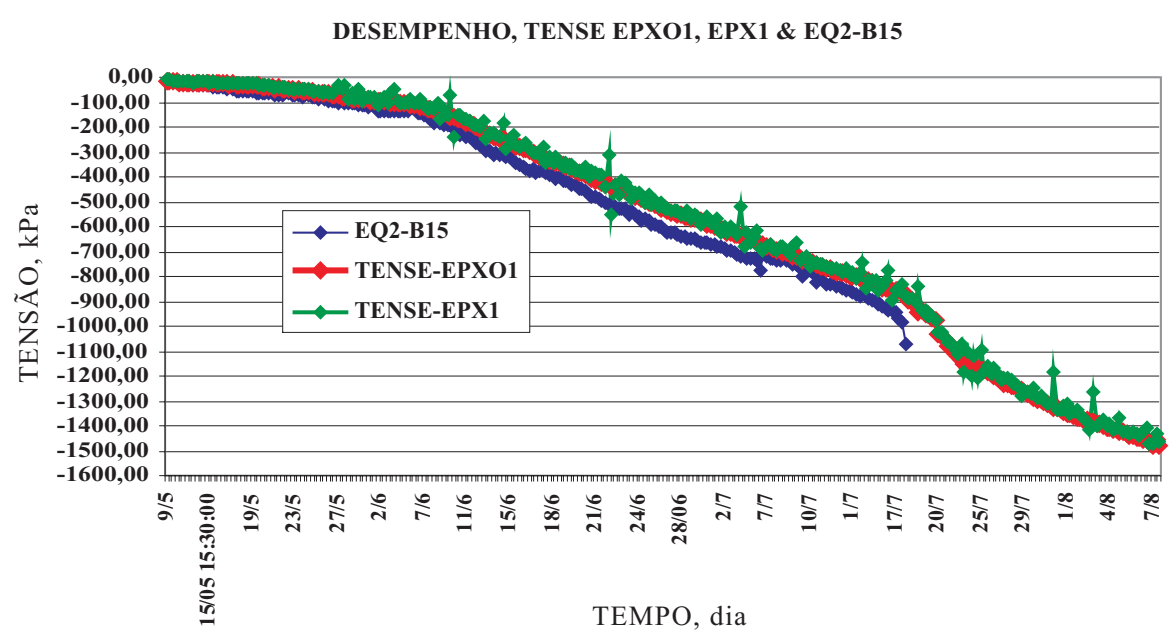

Figura 15. Resultados dos tensiômetros de tensão elevados, correlacionados com os do tensiômetro de equivalência ensaiado no lisímetro (tanque B). (Diene, 2004)

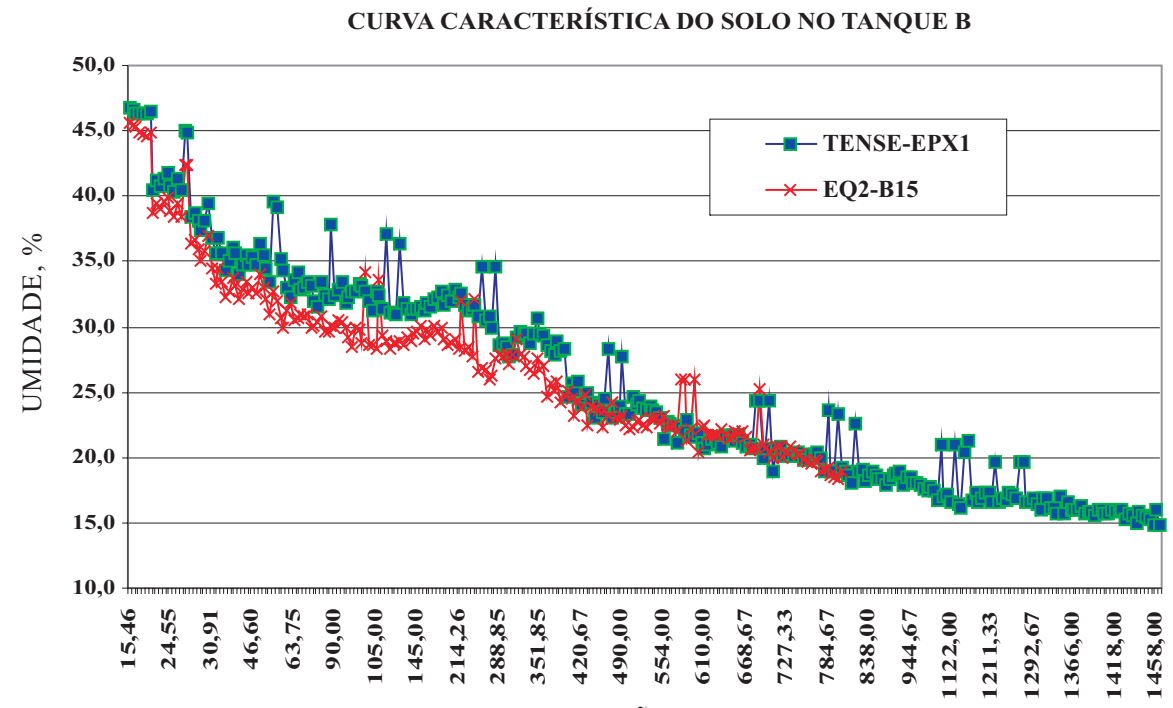

TENSÃO, $\mathrm{kPa}$

Figura 16. Aspecto da curva característica de retenção de umidade a partir dos dados dos tensiômetros de equivalência e TDR. Diene (2004)

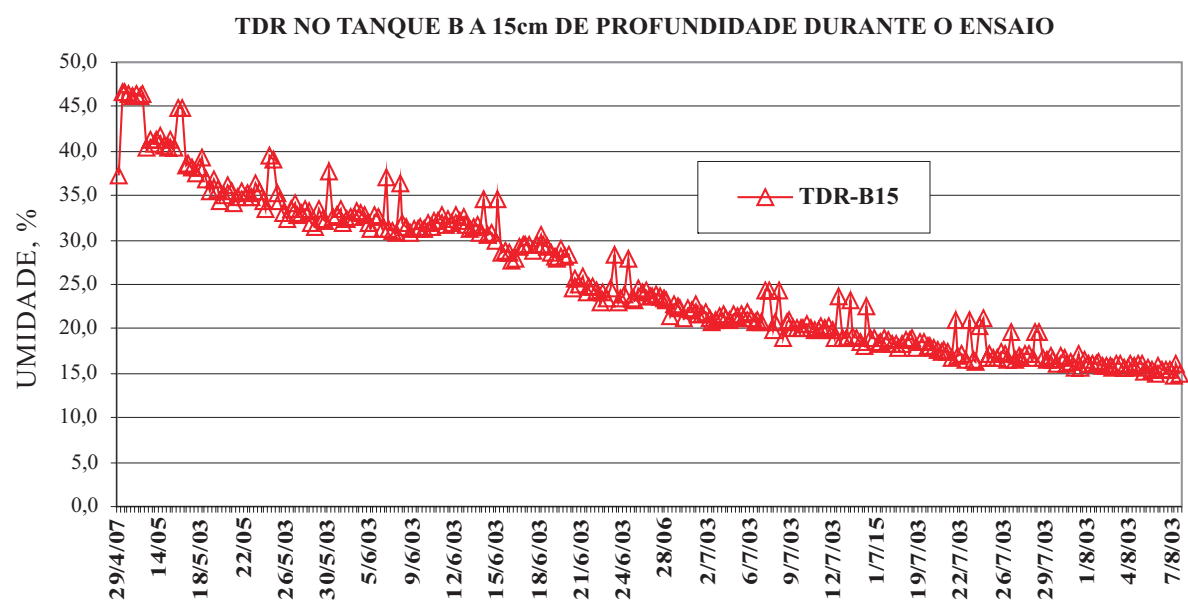

TEMPO, dia

Figura 17. Aspecto da variação de umidade a partir dos dados coletados pelo TDR-B15. Diene (2004) 


\section{DISCUSSÕES}

A medição, feita pelo TENSE-EPXO1, de valores acima do valor de pressão de entrada de ar já foi observada por outros pesquisadores, como Ridley \& Burland (1993) e Tarantino et al.(2000). Segundo Marinho \& Chandler (1995), esses valores poderiam ser atribuídos a uma certa eficiência osmótica da pedra porosa, e o tensiômetro estaria medindo tensão total em vez de tensão matricial. Outra justificativa possível para explicação de tal acontecimento pode ser também o fator de segurança dado pelo fabricante sobre o valor da pressão de entrada de ar da pedra porosa, que seria maior do que o indicado na pedra.

A diferença de tensão variável da ordem de 15 a $25 \mathrm{kPa}$ entre os tensiômetros TENSE-EPX1 e TENSEEPXO1 pode ser atribuída às oscilações na rede elétrica mensuradas pelo TENSE-EPX1, devido à sua alta sensibilidade (10,454 mV/FS). Um filtro de linha de melhor qualidade poderia evitar essas variações, as quais podem ser o motivo desse acréscimo de tensão matricial medida.

A diferença dos valores na faixa de medidas ( -100 a $-800 \mathrm{kPa}$, no caso do equitensiômetro) pode ser explicada pela imprecisão do equipamento observada pelo fabricante no seu manual. Contudo, essa variação não comprometeu as medições, já que as variações se mantiveram constantes com relação às faixas de medidas de tensões (Figura 15).

A similaridade do aspecto das curvas características do solo nas condições de campo e dos solos dos lisímetros indicaria a possibilidade de reproduzir condições de campo em laboratório com acurácia, delimitados os parâmetros de contorno.

A imediata resposta do tensiômetro durante a sua calibração com aplicação de cargas e o tempo de equalização das cargas aplicadas em menos de $30 \mathrm{~s}$ durante todo o processo de calibração e em todos os protótipos indicariam que o tempo de resposta do equipamento é adequado às leituras para as quais foi proposto, como se confirmou após instalação dos equipamentos nos lisímetros e imediata mensuração de dados no data-logger.

\section{CONCLUSÕES}

1. Os processos de saturação e calibração foram eficazes, tendo em vista que não se usaram altas pressões nem tempo de aplicação de carga duradora (dias) e que bastaram os ciclos de carga e descarga para a garantia de uma boa saturação, além da aplicação de vácuo e uso de água dearada.

2. Os resultados obtidos mostram que o protótipo desenvolvido pode ser empregado seja em medidas in situ, seja em laboratório, com a adaptação deste, por exemplo, às células de ensaios triaxiais e de adensamento.
3. Pode-se afirmar que a possibilidade de cavitação é dependente das condições térmicas e do estado de tensões no conjunto solo, pedra porosa, água e tensiômetro como um todo. Os fenômenos físicos da água, como forças de atração de Van der Waals, são perspectivas importantes no aprofundamento do estudo teórico dos diversos agentes no processo de cavitação, na medida de tensão em solos.

\section{AGRADECIMENTOS}

Os autores agradecem ao CNPq, à CAPES, à Fundação Volkswagen e ao Instituto de Pesquisas de Sistemas Ambientais da Universidade Osnabrück, Alemanha, pelo constante apoio a este projeto.

\section{NOTA}

O protótipo do tensiômetro utilizado como referência para o equipamento usado neste trabalho teve registro de patente solicitado no INPI sob o número 0403670-0 01/09/2004 pelos senhores Claudio Fernando Mahler e Eng. . Helcio Gonçalves de Souza.

\section{LITERATURA CITADA}

DELTA-T DEVICES, UK, Instruments for environmental and industrial measurement, 2000. Disponível em: http:/ www.delta-t.co.uk/products.html?product2005092818784

DIENE, A.A. Desenvolvimento de tensiômetros para sucção elevada, ensaiados em lisímetros de laboratório. Rio de Janeiro, Universidade Federal Rural do Rio de Janeiro, 2004. 173p. (Tese de Mestrado)

GUAN, Y. \& FREDLUND, D.G. Direct measurement of high soil suction. In: SIMPÓSIO SOLOS NÃO SATURADOS BRASILEIRO, 3., Rio de Janeiro, 1997. v.2. p.543-550.

GUAN, Y.; FREDLUND, D.G. \& GAN, J.K.M. Behavior of water subjected to high tensile stresses. :In: INTERNATIONAL CONFERENCE ON UNSATURATED SOILS, 2., Beijing, 1998. Proceedings. Beijing, International Academic Publishing, 1998. p.356-361.

HARVEY, E.N.; BARNES, D.K.; McELROY, W.D.; WHITELEY, A.H.; PEASE, D.C. \& COOPER, K.W. Bubble formation in animals.I Physical factors. J. Cell. Comparative Physiol., 24:1-22, 1944

KÖNIG, D.; JESSBERGER, H.L.; BOLTON, M.D.; PHILLIPS, R.; BAGGE, G.; RENZI, R. \& GARNIER, J. Pore pressure measurement during centrifuge model test: Experience of five laboratories. In: LEUNG, C.F.; LEE, F.H. \& TAN, T.S., eds INTERNATIONAL CONFERENCE CENTRIFUGE 94. Singapure, 1994. Proceedings. National University of Singapore, 1994. p.101-108. 
MARINHO, F.A.M. \& CHANDLER, R.J. Cavitation and the direct measurement of soil suction. In: INTERNATIONAL CONFERENCE ON UNSATURATED SOILS, 1., Paris, 1995. Proceedings. Paris, 1995.v.2. p.623-630.

PACHECO, A.C. Desenvolvimento de um novo tensiômetro para medidas de tensão acima de $1 \mathrm{Atm}$. Rio de Janeiro, Universidade Federal Rural do Rio de Janeiro, 2001. 93p.(Tese de Mestrado)

POROUS STONE - disponível em < http:// www.soilmoisture.com>

RIDLEY, A.M \& BURLAND, J.B. Discussion: Use of tensile strength of water for the direct measurement of high soil suction. Can. Geotechn. J., 36:178-180, 1999.

RIDLEY, A.M. Strength-suction-moisture content relationship for kaolin under normal atmospheric conditions. In: INTERNATIONAL CONFERENCE ON UNSATURATED SOILS, 1., Paris, 1995. Proceedings. Paris, 1995. v.2. p.645-651.

RIDLEY, A.M. The measurement of soil moisture suction. London, University of London, 1993. (Tese de Doutorado)
RIDLEY, A.M. \& BURLAND, J.B. A new instrument for measuring soil moisture suction. Geotechnique, 43:321324, 1993.

RIDLEY, A.M. \& BURLAND, J.B. Measurement of suction in materials which swell. Appl. Mechanics Rev., 48:727-732, 1995.

TABOR, D. Gases, liquids and solids. 2.ed. Cambridge, Cambridge University Press, 1979. 448p.

TAKE, W.A. \& BOLTON, M.D. A new device for the measurements of negative pore water pressure in centrifuge models. In: INTERNATIONAL CONFERENCE PHYSICAL MODELLING GEOTECHNICS, 2002, St. John's. Proceedings. Rotterdam, A. Balkema, 2002. p.89-94.

TARANTINO, A. \& MONGIOVİ, L. Design and construction of a tensiometer for direct measurement of matric suction. In: INTERNATIONAL CONFERENCE ON UNSATURATED SOILS, 3., Recife, 2002. Proceedings. Recife, 2002. p.319-324.

TARANTINO, A.; BOSCO G. \& MONGIOVÌ L. Response of the IC tensiometer with respect to cavitation. In: ASIAN CONFERENCE ON UNSATURATED SOILS, 2000, Singapure. Proceedings. Rotterdam, A. Balkema, 2000. p.309-314. 